\title{
Refinement-based Disintegration: An Approach to Re-representation in Relational Learning
}

\author{
Santiago Ontañón ${ }^{\mathrm{a}}$ and Enric Plaza ${ }^{\mathrm{b}}$ \\ ${ }^{\text {a }}$ Computer Science, Drexel University \\ Philadelphia 19104, PA, USA, \\ e-mail:santi@cs.drexel.edu \\ b IIIA, Artificial Intelligence Research Institute \\ CSIC, Spanish Council for Scientific Research \\ Campus UAB, 08193 Bellaterra, Catalonia (Spain), \\ e-mail:enric@iiia.csic.es
}

\begin{abstract}
We present a new approach lo learn from relational data based on re-representation of the examples. This approach, called property-based re-representation is based on a new analysis of the structure of refinement graphs used in ILP and relational learning in general. This analysis allows the characterization of relational examples by a set of multirelational patterns called properties. Using them, we perform a property-based re-representation of relational examples that facilitates the development of relational learning techniques. Additionally, we show the usefulness of rerepresentation with a collection of experiments in the context of nearest neighbor classification.
\end{abstract}

Keywords: Relational Learning, Re-representation, Refinement Operators, Feature Terms, Propositionalization

\section{Introduction}

Relational Machine Learning (RLM) studies how to design machine learning algorithms for domains where data is structured, or relational. In order to address this problem, in this paper we present a new approach lo learn from relational data based on re-representation of the examples. This approach, called property-based re-representation is based on a new analysis of the structure of refinement graphs used in Inductive Logic Programming (ILP) and relational learning in general. This analysis allows the characterization of relational examples by a set of multi-relational patterns called properties, with which we perform a property-based re-representation of relational examples that facilitates the development of relational learning techniques.

Our approach is based on the notion of refinement graphs [14], which are commonly used in ILP [15] and other areas, such as Description Logics, for performing inductive relational learning $[5,19,20]$. A refinement graph in the space of generalizations is built by defining a suitable refinement operator, which can specialize a given description to form more specific descriptions. Given the most general description that can be expressed in a given representation language, refinement operators can generate the whole space of expressions in such language by iteratively refining (i.e. specializing or generalizing) such description.

The approach and techniques presented here are, in principle, applicable to any representation language for which we can define a refinement operator that satisfies certain properties, namely they have to be complete and locally finite. For reasons of space an clarity, however, this paper will focus on a particular representation formalism, namely feature terms (presented in the next section), for which we will define its own refinement graph. Then, we present the notion of a property (a multi-relational pattern) of a relational example, and the disintegration operation, which splits a given relational example in a collection of properties. These properties can later be integrated again if need be to reconstruct the original example. Moreover, we introduce the way to re-represent an example as a set of properties and the building of a vocabulary of properties to represent examples in a given data set. The reason to present this re-representation is that it allows to use classical propositional machine learning techniques (with a small adaptation) to relational data, as discussed in Section 8. After showing the usefulness of our approach with an empirical evaluation in the context of nearest-neighbor classification, we discuss the relation of our approach with existing approaches such as propositionalization. The paper closes with related work and conclusions. 


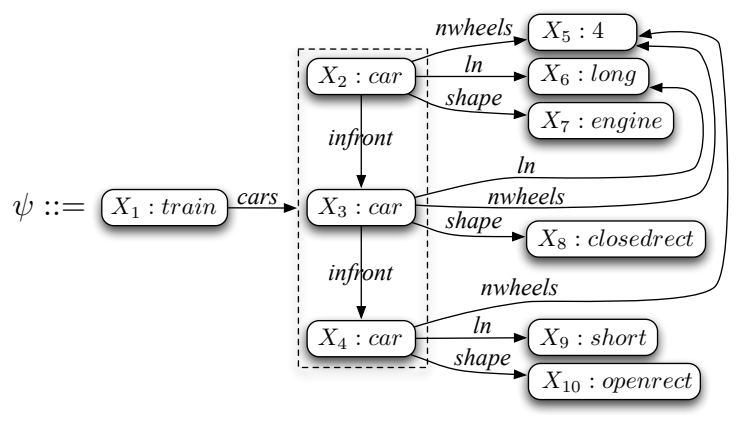

Fig. 1. A train represented as a feature term.

\section{Preliminaries}

This section introduces the formalism of feature terms, and the basic notions of refinement operators and graphs.

\subsection{Feature Terms}

Feature terms [2,7] (also called feature structures, or $\Psi$-terms) are a generalization of first-order terms, introduced in theoretical computer science to formalize object-oriented declarative languages. Feature terms correspond to a different subset of first-order logics than Description Logics. However, they have the same expressive power - only differing in their basic reasoning mechanisms [1].

Feature terms are defined by its signature: $\Sigma=$ $\langle\mathcal{S}, \mathcal{F}, \leq, \mathcal{V}\rangle . \mathcal{S}$ is a set of sort symbols, including $\perp$ representing the most general sort ("any"), and $\top$ representing the most specific sort ("none"); $\leq$ is an order relation inducing a single inheritance hierarchy in $\mathcal{S}$, and where $\perp \leq s \leq \top$ for each sort $s \in \mathcal{S}$. Specifically, $s \leq s^{\prime}$ means $s$ is more general than or equal to $s^{\prime}$, for any $s, s^{\prime} \in \mathcal{S}$ ("any" is more general than any $s$ which, in turn, is more general than "none"). $\mathcal{F}$ is a set of feature symbols, and $\mathcal{V}$ is a set of variable names. We define a feature term $\psi$ as,

$$
\psi::=X: s \quad\left[f_{1} \doteq \Psi_{1}, \ldots, f_{n} \doteq \Psi_{n}\right]
$$

where $\psi$ points to the root variable $X$ (that we will note as $\operatorname{root}(\psi)$ ) of sort $s ; X \in \mathcal{V}, s \in \mathcal{S}, f_{i} \in \mathcal{F}$, and $\Psi_{i}$ is either a variable $Y \in \mathcal{V}$, or a set of variables $\left\{X_{1}, \ldots, X_{m}\right\}$. When the value of some feature of two variables $X_{i} . f_{1}$ and $X_{j} \cdot f_{2}$ share some variable, i.e. $X_{i} \cdot f_{1} \cap X_{j} \cdot f_{2} \neq \emptyset$, we say that there is a variable equality.

An example feature term appears in Figure 1. It is a train (variable $X_{1}$ ) composed of three cars (variables
$X_{2}, X_{3}$ and $X_{4}$ ). This term has 10 variables, and one setvalued feature (its values enclosed in a dashed line): cars of $X_{1}$. There are also several variable equalities, e.g. equality $X_{3}$.infront $=X_{4}$. means that the car in front of car $X_{3}$ is car $X_{4}$. The set of variables of a term $\psi$ is $\operatorname{vars}(\psi)$, the set of features of a variable $X$ is fea$\operatorname{tures}(X)$, and $\operatorname{sort}(X)$ is its sort.

The basic relation over feature terms is subsumption (Б), i.e. whether a term is more general (or equal) than another $^{1}$.

Definition 1. (Subsumption) A feature term $\psi_{1}$ subsumes another one $\psi_{2}\left(\psi_{1} \sqsubseteq \psi_{2}\right)$ when there is a total mapping $m: \operatorname{vars}\left(\psi_{1}\right) \rightarrow \operatorname{vars}\left(\psi_{2}\right)$ such that:

1. $\operatorname{root}\left(\psi_{2}\right)=m\left(\operatorname{root}\left(\psi_{1}\right)\right)$, and

2. $\forall X \in \operatorname{vars}\left(\psi_{1}\right)$

(a) $\operatorname{sort}(X) \leq \operatorname{sort}(m(X))$, and

(b) $\forall f \in$ features $(X)$, where $X . f=\Psi_{1}$ and $m(X) \cdot f=\Psi_{2}$, we have that:

i. $\forall Y \in \Psi_{1}, \exists Z \in \Psi_{2}: m(Y)=Z$,

ii. $\forall Y, Z \in \Psi_{1}, Y \neq Z \Rightarrow m(Y) \neq m(Z)$

i.e. each variable in the set $\Psi_{1}$ is mapped to a variable in $\Psi_{2}$, and each different variable in $\Psi_{1}$ has a different mapping.

If $\psi_{1} \sqsubseteq \psi_{2}$ and $\psi_{2} \sqsubseteq \psi_{1}$, we say that they are equivalent: $\psi_{1} \equiv \psi_{2}$.

Subsumption induces a partial order over the set of all feature terms, i.e. the pair $\langle\mathcal{L}, \sqsubseteq\rangle$ is a poset, where $\mathcal{L}$ is the set of all feature terms that can be formed given a signature, and that contain the infimum $\perp$ and the supremum $T$ with respect to the subsumption order. The subsumption relation allows us to view the space of feature terms as a directed graph (called the subsumption graph) where nodes are feature terms and directed edges indicate subsumption.

The two basic operations over the subsumption graph are unification and antiunification.

Definition 2. (Unification) The unification $\psi_{1} \sqcup \psi_{2}$ of two terms $\psi_{1}$ and $\psi_{2}$ is the most general term subsumed by both. A term $\psi$ is called the unifier whenever:

$$
\begin{aligned}
\psi_{1} \sqcup \psi_{2}=\psi: & \left(\psi_{1} \sqsubseteq \psi \wedge \psi_{2} \sqsubseteq \psi\right) \wedge \\
& \left(\nexists \psi^{\prime} \sqsubset \psi: \psi_{1} \sqsubseteq \psi^{\prime} \wedge \psi_{2} \sqsubseteq \psi^{\prime}\right)
\end{aligned}
$$

\footnotetext{
${ }^{1}$ In description logics notation, subsumption is written in the reverse order since it is seen as "set inclusion" of their interpretations. In ML terms, $A \sqsubseteq B$ means that $A$ is more general than $B$, while in description logics it has the opposite meaning.
} 

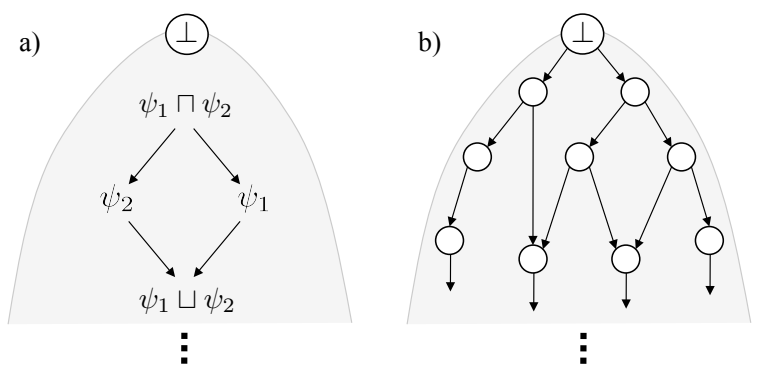

Fig. 2. a) Illustration of the unification and antiunification concepts, b) refinement graph.

When two terms have contradictory information then they have no unifier —equivalently we write $\psi_{1} \sqcup \psi_{2}=$ $\top$.

The antiunification $\left(\psi_{1} \sqcap \psi_{2}\right)$ of two terms $\psi_{1}$ and $\psi_{2}$ is defined as their least general generalization:

Definition 3. (Antiunification) The antiunification $\psi_{1} \sqcap$ $\psi_{2}$ of two terms $\psi_{1}$ and $\psi_{2}$ is the most specific term that subsumes both. The term is called the antiunifier.

$$
\begin{aligned}
\psi_{1} \sqcap \psi_{2}=\psi: & \left(\psi \sqsubseteq \psi_{1} \wedge \psi \sqsubseteq \psi_{2}\right) \wedge \\
& \left(\nexists \psi^{\prime} \sqsupset \psi: \psi^{\prime} \sqsubseteq \psi_{1} \wedge \psi^{\prime} \sqsubseteq \psi_{2}\right)
\end{aligned}
$$

Both unification and antiunification are operations over the subsumption graph: antiunification finds the most specific common "parent" (generalization); unification finds the most general common "descendant" (specialization). Moreover, unification and antiunification are not unique for the subsumption graph of feature terms. This means that the refinement graph of feature terms is not a lattice. Figure 2.a illustrates unification and antiunification, showing the entire space of feature terms (with the most general term, $\perp$, at the top) and arrows indicating subsumption.

\subsection{Refinement Operators}

Let us now define the notion of refinement operator (for a more in depth discussion of refinement operators, see [14]), which can be used to navigate the subsumption graph, and, in general, any partially-ordered or quasi-ordered set. In the remainder of this article we will consider only the case of partially-ordered sets (i.e. in which two terms which subsume each other are considered equivalent: $\psi_{1} \equiv \psi_{2}$ ).

Definition 4. A downward refinement operator $\rho$ over a partially-ordered set $(\mathcal{L}, \sqsubseteq)$ is a function such that $\forall \psi \in \mathcal{L}: \rho(\psi) \subseteq\left\{\psi^{\prime} \in \mathcal{L} \mid \psi \sqsubseteq \psi^{\prime}\right\}$
Definition 5. An upward refinement operator $\gamma$ over a partially-ordered set $(\mathcal{L}, \sqsubseteq)$ is a function such that $\forall \psi \in \mathcal{L}: \gamma(\psi) \subseteq\left\{\psi^{\prime} \in \mathcal{L} \mid \psi^{\prime} \sqsubseteq \psi\right\}$.

In other words, upward refinement operators generate terms which are more general, whereas downward refinement operators generate terms which are more specific. Typically, the symbol $\gamma$ is used to symbolize upward refinement operators, and $\rho$ to symbolize either a downward refinement operator, or a refinement operator in general. The following properties of refinement operators are considered desirable:

1. A refinement operator $\rho$ is locally finite if $\forall \psi \in$ $\mathcal{L}: \rho(\psi)$ is finite.

2. A downward refinement operator $\rho$ is complete if $\forall \psi_{1}, \psi_{2} \in \mathcal{L} \mid \psi_{1} \sqsubseteq \psi_{2}: \psi_{2} \in \rho^{*}\left(\psi_{1}\right)$.

3. An upward refinement operator $\gamma$ is complete if $\forall \psi_{1}, \psi_{2} \in \mathcal{L} \mid \psi_{1} \sqsubseteq \psi_{2}: \psi_{1} \in \rho^{*}\left(\psi_{2}\right)$.

4. $\rho$ is proper if $\forall \psi_{1}, \psi_{2} \in \mathcal{L} \psi_{2} \in \rho\left(\psi_{1}\right) \Rightarrow \psi_{1} \not \equiv$ $\psi_{2}$.

where $\rho^{*}$ stands for the transitive closure of a refinement operator. Intuitively, locally finiteness means that the refinement operator is computable, completeness means that all the terms in $\mathcal{L}$ can be generated by refinement, and properness means that a refinement operator does not generate elements which are equivalent to a given term $\psi$. A refinement operator is ideal when is locally finite, complete and proper. When a refinement operator is locally finite, complete and proper, we say that it is ideal. Other interesting properties, like minimality [5], have been discussed in the literature but are not relevant for this paper.

The refinement graph is the graph where each node is one term, and there is a link between two terms when one is a refinement of the other. Figure 2.b illustrates a refinement graph, where more general terms are drawn at the top, and arrows indicate specialization refinement. Notice that the refinement graph is contained in the subsumption graph.

\section{Properties and Disintegration}

This section presents the disintegration operation, which disintegrates a given term (which might represent an instance or a generalization) into a set of properties. We introduced the idea of disintegration in our past work for the purposes of similarity assessment (a more informal definition can be found in [18]). The intuitive idea of disintegration is that we want to transform a term into a set containing the most basic pieces 
of information the original term contains. For example, in the train shown in Figure 1, one property is that the train has 3 cars, another is that the first car has 4 wheels, another is that the number of wheels of the second car is the same as in the first car, etc. The disintegration operation provides with a formal and principled way to perform this process, based upon the existence of a refinement operator.

\subsection{Properties}

The disintegration operation defined in this section specifically splits a given term in a collection of smaller terms, called properties, representing precisely these most primitive pieces of information. We will also show that, under certain assumptions, those properties can be integrated again to reconstruct the original example.

Before defining the properties of a term, we will first define the remainder of a generalization refinement operator.

Definition 6. (Remainder) Given a term $\psi_{2} \in \gamma\left(\psi_{1}\right)$, where $\gamma$ is a generalization refinement, the remainder $r\left(\psi_{1}, \psi_{2}\right)$ of such generalization is a term $\pi$ such that $\pi \sqcup \psi_{2} \equiv \psi_{1}$ and $\nexists \psi^{\prime} \in \mathcal{L}$ such that $\psi^{\prime} \sqsubset \pi$ and $\psi^{\prime} \sqcup$ $\psi_{2} \equiv \psi_{1}$.

That is to say, the remainder of a generalizing refinement $\gamma$ from $\psi_{1}$ to $\psi_{2}$ is the most general term $\pi$ such that when unified with the generalization $\psi_{2}$ obtains back the original term $\psi_{1}$. We will call this remainder $\pi$ a property of $\psi_{1}$. Notice that the remainder is the most general term that captures which is the "property" that $\psi_{1}$ has and that is not present in $\psi_{2}$, i.e. the informational content that the generalization operator removed. Figure 3 illustrates this idea, where a train $\psi_{1}$ is generalized with a refinement operator to $\psi_{2}$ : the property subtracted is the fact that the car of that train has 2 wheels. Notice that a property is, in general, a multirelational pattern, with relations cars and nwheels in the property of Fig. 3. The remainder of a specialization refinement $\rho$ can be defined similarly.

\subsection{Disintegration}

Now, if we iterate this generalization refinement over the resulting term and keep generalizing it, we will obtain a collection of properties as remainders of each step. In the end, the iterative generalization process will reach $\perp$, the empty term, and we will have a collection of properties satisfied by the initial term.
This is the intuitive idea of term disintegration: generalize a term repeatedly until reaching $\perp$ while collecting a property at each step by getting the remainder of the generalization operation. Let us formally define such process.

Definition 7. (Refinement Path) A finite sequence of terms $\left(\psi_{1}, \ldots, \psi_{n}\right)$ is a refinement path $\psi_{1} \stackrel{\rho}{\rightarrow} \psi_{n}$ between two terms $\psi_{1}$ and $\psi_{n}$ when for each $1 \leq i<n$, $\psi_{i+1} \in \rho\left(\psi_{i}\right)$. The same definition applies for the generalization refinement operator: $\psi_{n} \stackrel{\gamma}{\rightarrow} \psi_{1}$.

Definition 8. (Disintegration) Given a finite refinement path $p=\psi_{1} \stackrel{\gamma}{\rightarrow} \perp$ consisting of a sequence of terms $\left(\psi_{1}, \ldots, \psi_{n}\right)$, where $\psi_{n}=\perp$, the set $D_{p}\left(\psi_{1}\right)=$ $\left\{r\left(\psi_{i}, \psi_{i+1}\right)\right\}_{1 \leq i<n}$ is a disintegration of the term $\psi_{1}$.

That is to say, $D_{p}\left(\psi_{1}\right)$ is the set of remainders resulting from each generalization step performed by the refinement operator $\gamma$ in the path $p$ from $\psi_{1}$ to $\perp$.

Given a refinement path $p=\psi \stackrel{\gamma}{\rightarrow} \perp$, and having in mind that refinement operators represent the most fine-grained steps in which terms can be specialized or generalized, the remainders obtained from such paths correspond to the most primitive pieces of information (relative to the refinement operator) contained in a term $\psi$. Therefore, the disintegration of a term is a process that breaks up a term into its most constituent and primitive pieces of information (with respect to a particular language); each one of these pieces of information is also represented as a term, and this is what we call a property.

Lemma 1. If $\psi_{1} \stackrel{\gamma}{\rightarrow} \perp$ is a refinement path consisting of a sequence of terms $\left(\psi_{1}, \ldots, \psi_{n}=\perp\right), \gamma$ is proper, and $1 \leq i<j<n$, then $r\left(\psi_{i}, \psi_{i+i}\right) \nsubseteq r\left(\psi_{j}, \psi_{j+i}\right)$.

Proof. By Definition 7, we know that if $\gamma$ is proper, then $\psi_{k+1} \sqsubset \psi_{k}$ for $1 \leq k<n$. Now, let $\psi_{a}=$ $r\left(\psi_{i}, \psi_{i+i}\right)$, and $\psi_{b}=r\left(\psi_{j}, \psi_{j+i}\right)$. By Definition 6, $\psi_{j} \in \psi_{j+1} \sqcup \psi_{b}$, and thus $\psi_{b} \sqsubseteq \psi_{j}$, since $j>i$, we know that $\psi_{j} \sqsubseteq \psi_{i+1}$, and thus $\psi_{b} \sqsubseteq \psi_{i+1}$. Now, let us assume $\psi_{a} \sqsubseteq \psi_{b}$. Since $\psi_{a} \sqsubseteq \psi_{b} \sqsubseteq \psi_{i+1}$, we know that $\psi_{i+1} \sqcup \psi_{b}=\psi_{i+1}$, and $\psi_{i+1} \sqcup \psi_{a}=\psi_{i+1}$. Since $\gamma$ is proper, we know that $\psi_{i} \neq \psi_{i+1}$. Therefore $\psi_{i} \notin$ $\psi_{i+1} \sqcup \psi_{a}$, which is in contradiction with Definition 6 , and thus our assumption that $\psi_{a} \sqsubseteq \psi_{b}$ has to be false.

The disintegration of a term $\psi$ is described in Algorithm 1. Given a term $\psi$, and a generalization refinement operator $\gamma$, the algorithm proceeds iteratively, generalizing $\psi$ using $\gamma$, until $\perp$ is reached. At each it- 


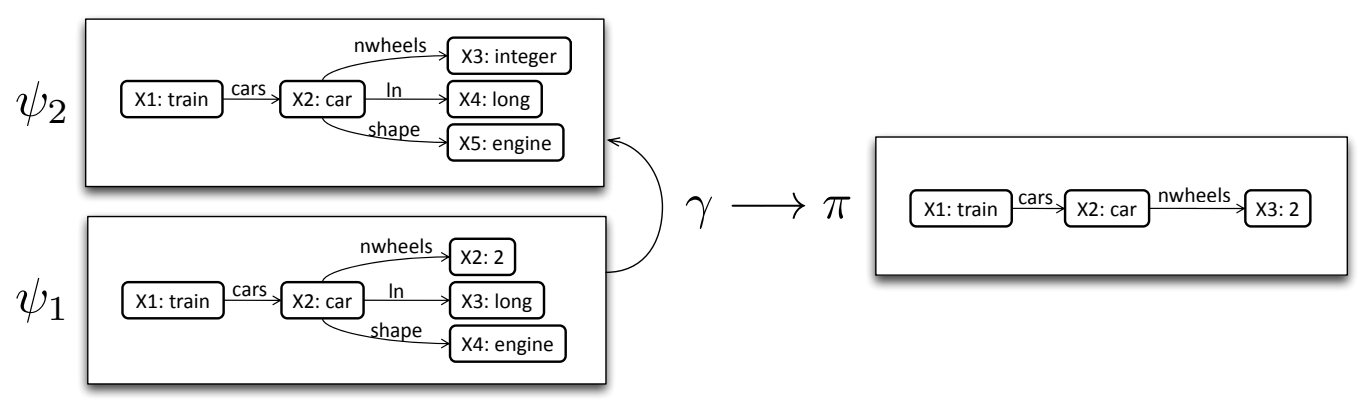

Fig. 3. A refinement operator $\gamma$ that generalizes $\psi_{1}$ into $\psi_{2}$ by subtracting a piece of information $\pi$ called the remainder of the refinement.

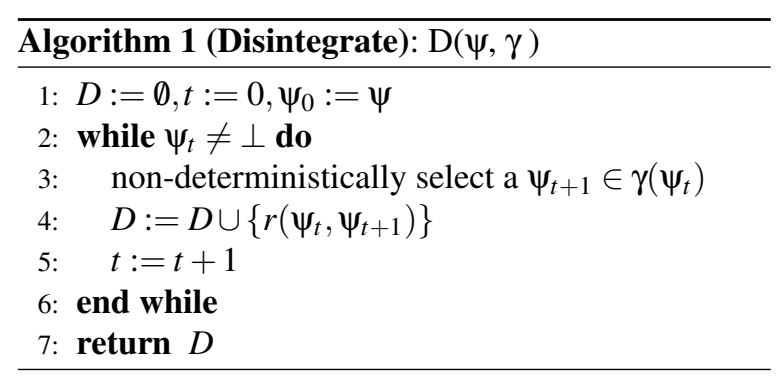

eration $t$ of the algorithm, a new generalization $\psi_{t+1}$ is generated by taking one of the generalizations (one can be chosen at random) generated by $\gamma$ from the current term $\psi_{t}$. Then, the property set $D$ is expanded by adding the remainder $r\left(\psi_{t}, \psi_{t+1}\right)$ of generalizing $\psi_{t}$ into $\psi_{t+1}$. When $\perp$ is reached, the algorithm returns the set $D$ containing all the properties generated so far, corresponding to a disintegration of the term $\psi$.

Notice that step 3 in Algorithm 1 is non-deterministic, since any refinement can be chosen. This means, that depending on the choice of refinements (i.e. depending on the refinement path), different disintegrations might be obtained. It can be shown that under certain conditions (e.g. the refinement graph being a lattice), then the choice of refinements is irrelevant, since all of them will result in the same disintegration. However, in general, this is not true. As part of our future work, we plan to investigate the possibility of different strategies for making this non-deterministic choice, which produce unique disintegrations.

Figure 4 shows an example of the disintegration process, where a simple train represented as a feature term (top half) has been disintegrated into properties (bottom half). Disintegration extracted 14 properties from this train, since the refinement path used had 14 generalization steps. Notice how the properties respect Lemma 1, i.e. the properties generated earlier cannot be more general than the properties generated later.

The integration of a property set is the opposite process of disintegration.
Definition 9. (Integration) The integration of a set of properties $D(\psi)$, obtained via disintegrating a term $\psi$ is defined as:

$$
\operatorname{integrate}(D(\psi))=\bigsqcup(D(\psi))
$$

In other words, integration is defined as the unification of all properties of a disintegrated term $\psi$. Given that unification of feature terms is not unique, there might be multiple different integrations of a given set of properties. It can be easily seen that one of the different integrations of a disintegrated term is equivalent to (in the sense of ' $\equiv$ ') the original term.

Lemma 2. One of the unifications of all the properties of a term $\psi$ is exactly the term $\psi$, i.e. $\psi \in \bigsqcup(D(\psi))$. When unification is unique, then $\psi=\bigsqcup(D(\psi))$.

Proof. Let $\psi_{1}$ be a term that when disintegrated using the refinement path $\left(\psi_{1}, \ldots, \psi_{n}=\perp\right)$ yields the properties $\left(r_{1}, \ldots, r_{n-1}\right)$. Definition 6 (Remainder) ensures that $\psi_{i+1} \sqcup r_{i}=\psi_{i}$ (or that $\psi_{i}$ is one of the unifications if $\psi_{i+1} \sqcup r_{i}$ is not unique). Let us consider first the case when unification is unique. Iteratively unifying the properties in the reverse order in which they were generated, we can reconstruct the refinement path: $\psi_{n-1}=r_{n-1}, \psi_{n-2}=r_{n-1} \sqcup r_{n-2}, \psi_{n-3}=\left(r_{n-1} \sqcup\right.$ $\left.r_{n-2}\right) \sqcup r_{n-3}$, etc. Thus, $\psi_{1}=\bigsqcup_{i=n-1 \ldots 1} r_{i}$, which is precisely $\bigsqcup(D(\psi))$. When unification is not unique, we know by Definition 6 that: $\psi_{n-1}=r_{n-1}, \psi_{n-2} \in$ $r_{n-1} \sqcup r_{n-2}, \psi_{n-3} \in\left(r_{n-1} \sqcup r_{n-2}\right) \sqcup r_{n-3}$, etc. Thus, $\psi_{1} \in \bigsqcup_{i=n-1 \ldots 1} r_{i}$, which is precisely $\bigsqcup(D(\psi))$.

If another representation formalism, different from feature terms, were to be used where the refinement graph was a lattice, then integration would generate a single term, which would be the same as the original example. This is interesting, since it shows that not too much information is lost when disintegrating a term. 


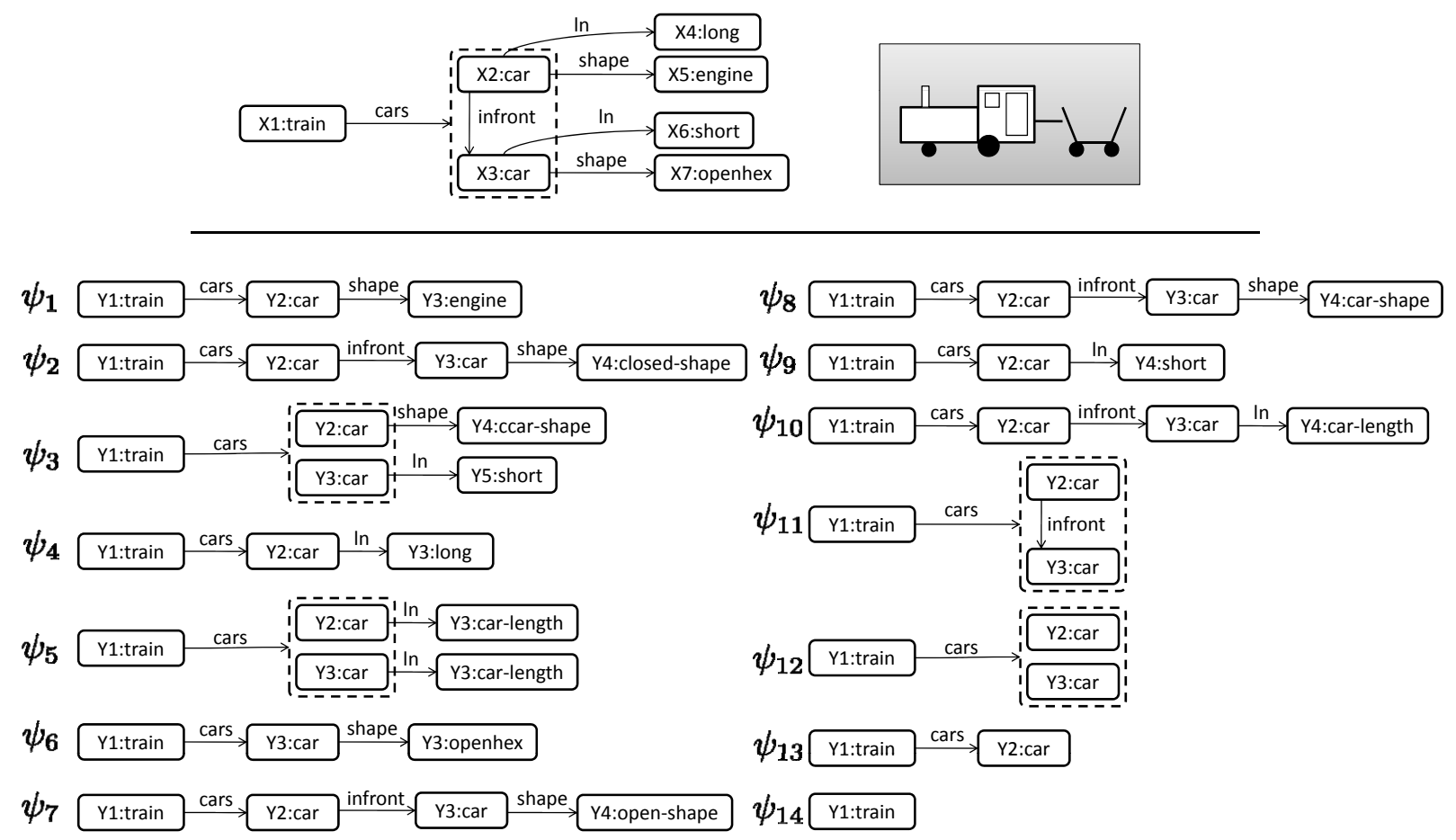

Fig. 4. An example feature term disintegrated into properties using Algorithm 1.

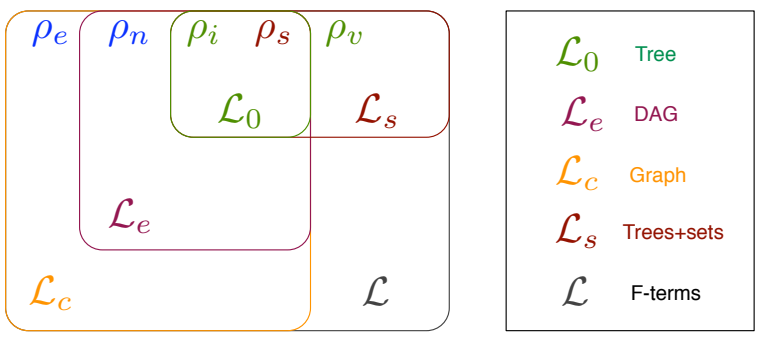

Fig. 5. Several sublanguages of feature terms generated by different refinement operators (shown as $\rho$ symbols on the top and defined in [18]).

\section{Re-representation with a Taxonomic Vocabulary}

The possibility of defining different refinement operators that generate different subsets of a given representation language opens the possibility of defining several different sublanguages of different expressive power and complexity. Consequently, the disintegration of the examples in a given training set would yield sets of properties in the particular sublanguage corresponding to the refinement operator being used. For example, Figure 5 shows the sublanguages defined in [18] for feature terms. Summarily, $\mathcal{L}$ is the complete feature term language as defined above; $\mathcal{L}_{0}$ contains all the feature terms that do not have any set-valued feature or any variable equality; $\mathcal{L}_{e}$ contains all the terms that do not have any set-valued feature or any circular variable equality (non-circular variable equalities are allowed); $\mathcal{L}_{c}$ is a super set of $\mathcal{L}_{e}$ which allows terms with circular variable equalities; and $\mathcal{L}_{s}$ is a super set of the base language $\mathcal{L}_{0}$ which allows set-valued features. These languages are generated using a different set of refinement operators (included in Appendix A).

The disintegration operation gives us the capability of re-representing examples in a vocabulary composed of properties of those examples.

Definition 10. (Vocabulary) A vocabulary $\mathbf{V}$ of properties for a set of examples $E=\left\{e_{1}, \ldots, e_{n}\right\}$ is a subset $\mathbf{V} \subseteq \bigcup_{i=1, \ldots, n} D\left(e_{i}\right)$

Definition 11. (Taxonomic Vocabulary) $A$ taxonomic vocabulary of a set properties $\mathbf{V}$ is the preorder $\langle\mathbf{V}, \sqsubseteq\rangle$, where $\sqsubseteq$ is the subsumption relation between properties.

Notice that the union of properties means that there will be no "repeated" properties in $\mathbf{V}$-i.e. if two properties are equivalent only one is in $\mathbf{V}$. Let us now show how a taxonomic vocabulary allows us to re-represent the set of examples $E=\left\{e_{1}, \ldots, e_{n}\right\}$ as a binary matrix. 


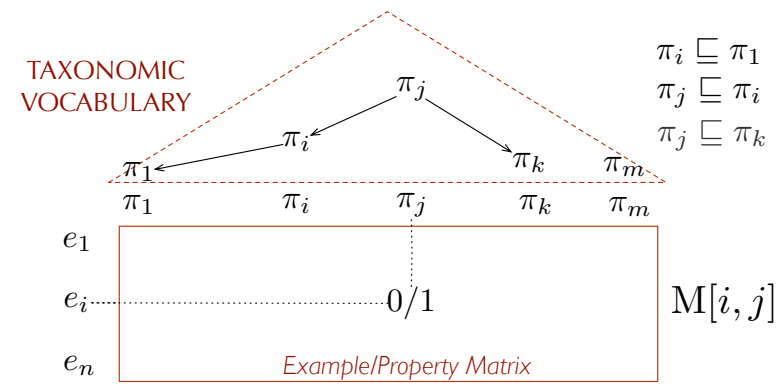

Fig. 6. Taxonomic vocabulary of properties to re-represent examples and the E/M matrix.

Definition 12. (Re-representation) A re-representation of a set of examples $E=\left\{e_{1}, \ldots, e_{n}\right\}$ with a taxonomic vocabulary $\langle\mathbf{V}, \sqsubseteq\rangle$, where $\mathbf{V}=\left\{\pi_{1}, \ldots, \pi_{m}\right\}$, is a $n \times m$ binary matrix $\mathbf{M}$ where

$$
\mathbf{M}[i, j]=\left\{\begin{array}{l}
1 \Leftrightarrow \pi_{j} \sqsubseteq e_{i} \\
0 \Leftrightarrow \pi_{j} \not \subset e_{i}
\end{array}\right.
$$

We call $\mathbf{M}$ the Example/Property (or E/P) matrix, as shown in Fig. 6.

Definition 13. (Example Re-representation) The rerepresenation of an example $e_{i}$ in a taxonomic vocabulary $\langle\mathbf{V}, \sqsubseteq\rangle$ is a Boolean vector $R\left(e_{i}\right)=\left(b_{1}, \cdots, b_{m}\right)$, where $b_{i} \in\{0,1\}$ such that $b_{j}=1$ whenever $\pi_{j} \sqsubseteq e_{i}$ and $b_{j}=0$ otherwise. Notice that each row of the $E / P$ matrix is an example re-representation.

Thus, given a dataset $E$, we can re-represent it by first disintegrating each of the examples in $E$, and then computing the E/P matrix. Moreover, a vocabulary $\mathbf{V}$ may also be built using only a subset of all the available examples in $E$. A simple way to do that is sampling the examples to be disintegrated. Let $\Sigma(E, \tau)$ be a sampling method (such as SRS or class-stratified sampling) that returns a $\tau$ percent of $E$; the corresponding vocabulary is $\mathbf{V}_{\Sigma(E, \tau)}=\bigcup_{e_{i} \in \Sigma(E, \tau)} D\left(e_{i}\right)$. For large data sets, sampling the examples to collect the properties for a vocabulary clearly diminishes computational cost, as long as the $\tau$ percent examples allow us to build a vocabulary $\mathbf{V}$ that is representative and satisfactory for the purposes at hand.

There are further operations that may reduce the size of a vocabulary $\mathbf{V}$. For instance, one may determine a reduced $\mathbf{V}^{\prime} \subseteq \mathbf{V}$ by removing properties in $\mathbf{V}$ that either (a) subsume all (or almost all) examples in $E$, and (b) subsume only one (or very few) examples in $E$.

Re-representation is a process that maps objects described in a formalism to descriptions on another for- malism, often because this second formalism is more adequate for some specific form of reasoning or inference (e.g. analogical reasoning [9]). A related notion is that of propositionalization in relational learning and ILP [12]; from our viewpoint, propositionalization is a specific kind of re-representation where relational representation of objects is mapped into a propositional language. In our approach, however, objects represented as feature terms are mapped not onto propositions but onto sets of feature terms (called properties). These feature terms (properties) constitute a partially ordered vocabulary whose elements are not simple propositions, since they have a strong structure based on subsumption. Moreover, properties are also related to the objects (examples) by subsumption.

Let us now analyze the structure of taxonomic vocabularies that are generated for different data sets.

\section{Structure of Taxonomic Vocabularies}

The disintegration operation (that we use for rerepresentation) maps a given relational example (represented, for example, as a feature term) to a set of properties. Moreover, these properties are not completely independent, but are related to each other via the subsumption relation. For example, in Figure 4, property $\psi_{14}$ subsumes property $\psi_{13}$. In a similar way, a taxonomic vocabulary $\mathbf{V}$ is not a plain set, but has internal structure (see Definition 11). This section illustrates the structure of these vocabularies for different types of datasets.

Figure 7 shows the set of 88 properties resulting from disintegrating one of the examples in the Demospongiae dataset. Demospongiae is a relational dataset containing descriptions of marine sponges. These descriptions are tree-like structures, and language $\mathcal{L}_{S}$ is enough to represent them (i.e. a feature term representing a sponge might have sets, but no variable equalities). If we disintegrate one of these sponges and create a graph where each node is one of the resulting properties and edges represent subsumption relations, we obtain a graph similar to the one shown in Figure 7 . We call this the property graph of a given feature term. Notice that since subsumption is transitive, if a property $\psi_{1}$ subsumes a property $\psi_{2}$, which subsumes a property $\psi_{3}$, we only draw edges between $\psi_{1}$ and $\psi_{2}$ and between $\psi_{2}$ and $\psi_{3}$; we do not include the edge between $\psi_{1}$ and $\psi_{3}$, which is implicit. Property graphs for feature terms represented by languages $\mathcal{L}_{0}$ and $\mathcal{L}_{S}$ result in trees, where the root is the most gen- 


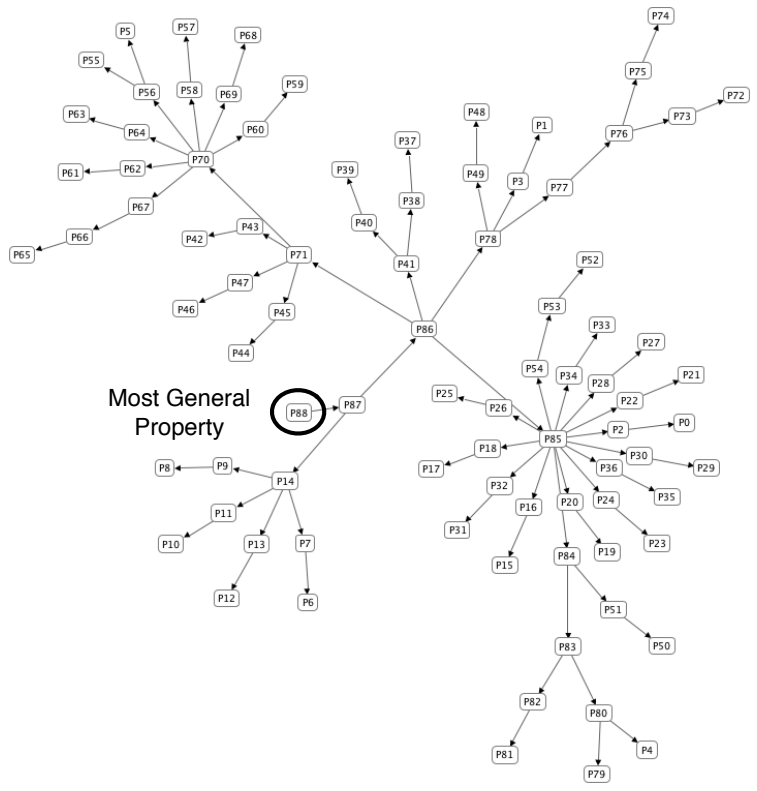

Fig. 7. The set of properties resulting from disintegrating an example in the Demospongiae data set. Arrows indicate subsumption.

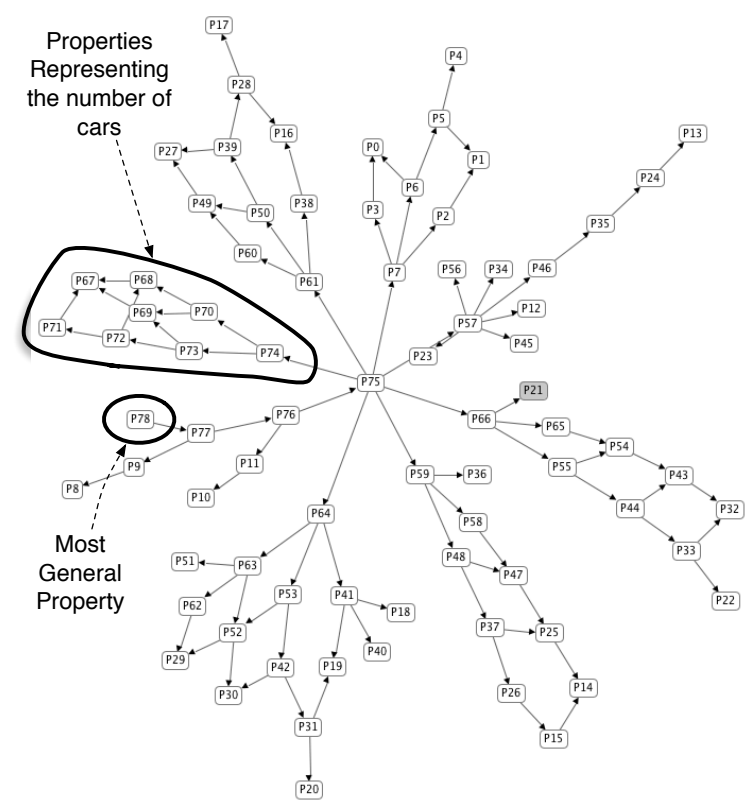

Fig. 8. The set of properties resulting from disintegrating an example in the Trains data set. Arrows indicate subsumption.

eral property. The same is true for the complete Taxonomic vocabularies generated for datasets that can be represented with these languages.

Figure 8 shows the property graph resulting from disintegrating an example in the Trains data set (where each train is represented similarly as the train in Figure 1). To represent this dataset, we need language $\mathcal{L}_{e}$ (we can have variable equalities, but not circular ones). The resulting structure of the property graph is a DAG (directed acyclic graph). Moreover, we can see that the resulting DAG is organized by smaller clusters of properties; in Figure 8, we have highlighted one of these clusters, containing all the properties that represent the number of cars in the train, and their relationships (which one is in front of which).

Notice that, by construction, the different properties in the re-representation of examples are not independent, as typically assumed by machine learning algorithms. Thus, it is interesting to know the structure of the property graph associated with the Taxonomic vocabularies for a given representation language, since this structure is tightly related with the dependencies between attributes. If we re-represent examples as binary vectors, the binary features that are related via subsumption are not independent. This information can be exploited for example for feature selection, or for increasing the efficiency of algorithms like decision tree inducers (ID3, C4.5, FOIL, etc.), since once a feature is selected, all of the features subsumed by it can be discarded in one of the subbranches of the tree. As part of our future work, we want to explore new algorithms that can further exploit this structure during learning.

\section{Experimental Results}

In order to evaluate our re-representation ideas, we performed a two sets of experiments. In the first experiment, we compared the performance of a nearestneighbor classifier using distance measures based on our re-representation versus a set of other relational distance measures that work directly over the relational representation of the examples. In the second experiment, we experimented with the effect that creating taxonomic vocabularies of different sizes has on performance.

We used three datasets, representing three levels of complexity: Soybean is a propositional data set (representable using $\mathcal{L}_{0}$ ) from the UCI machine learning repository consisting of 307 cases. Demospongiae is a relational dataset, where each example can be represented as a tree; it has 503 examples pertaining to 7 different classes (we used also a subset of 280 examples and 3 different classes, typically used in the literature for comparison purposes). Trains is a relational 
Table 1

Classification accuracy (in percentage) measured using a leave-oneout method for different distance/similarity measures.

\begin{tabular}{|c||c|c||c|c|c|c|}
\hline \multicolumn{1}{|c||}{} & \multicolumn{2}{|c||}{ Using re-representation } & \multicolumn{4}{c|}{ Relational Measures } \\
\hline & Jaccard & Euclidean & $S_{\lambda}$ & SHAUD & RIBL & Kashima \\
\hline Soybean-307 & 91.21 & 91.21 & 91.53 & 91.53 & 91.53 & 92.18 \\
\hline Demospongiae-280 & 95.00 & 93.47 & 95.00 & 95.71 & 91.67 & 90.71 \\
\hline Demospongiae-503 & 90.66 & 89.26 & 89.66 & 88.27 & 88.93 & 83.10 \\
\hline Trains-1000 & 90.70 & 90.40 & 84.80 & - & 67.10 & 63.90 \\
\hline
\end{tabular}

dataset, generated using Muggleton's train generator [17]. It contains 1000 trains belonging to 2 different classes; trains are represented as graphs.

We experimented with two different distance measures for the nearest-neighbor classifier: Jaccard and Euclidean. These two measures are defined as measures that compare two rows, $i$ and $j$ (representing two instances), of the re-representation matrix $\mathbf{M}$, as follows (assuming we have $n$ examples and $m$ properties in the vocabulary):

- Jaccard distance: typically defined to measure the difference between sets as the size of their intersection divided by the size of their union, can be computed from the matrix $\mathbf{M}$ as:

$$
d_{J}(i, j)=\frac{\sum_{k=1 \ldots m} M[i, k] M[j, k]}{\sum_{k=1 \ldots m} M[i, k]+M[j, k]-M[i, k] M[j, k]}
$$

\section{- Euclidean distance:}

$$
d_{E}(i, j)=\sqrt{\sum_{k=1 \ldots m}(M[i, k]-M[j, k])^{2}}
$$

The results from our first experiment can be seen on Table 1. We compared the performance of our rerepresentation against four other distance and similarity measures from the literature:

- $S_{\lambda}$ [18]: is a relational similarity measure based on computing the antiunification of the two examples, and then measuring its size (the larger the antiunification, the more similar the two examples).

- SHAUD [3]: is a similarity measure defined over feature terms. SHAUD is only defined for terms that can be represented as trees, and thus, it cannot be applied to the trains dataset.

- RIBL [8]: is a relational similarity measure defined over horn clauses. In order to compare against this measure, we converted all our datasets to Horn clauses, using the same procedure as [18].
- Kashima [10]: is a graph-kernel based on random walks. It requires the conversion of the examples to labelled graphs, which can be done trivially.

As Table 1 shows, using our re-representation achieves similar (and sometimes better) performance than using complex relational similarity measures. This indicates that our re-representation does not lose any significant information, at least in the datasets used for this study. Moreover, as any propositionalization technique, it has the advantage that once a dataset has been re-represented, applying propositional machine learning techniques is much more efficient.

Table 2 show the results we obtained disintegrating a different portion of examples $(\tau)$ of the dataset. Results were obtained using a leave-one-out procedure. As Table 2 shows, performance degrades very slowly when disintegrating smaller and smaller portions of a regular dataset. For example, we observed no difference in performance in any dataset when creating the Taxonomic Vocabulary by disintegrating only $40 \%$ of the examples in the dataset. Moreover, in some datasets, such as Trains, performance did not degrade at all even when only disintegrating a $1 \%$ of the examples in the dataset. This is because just disintegrating a small sample of examples in the dataset is enough for obtaining a good sample of binary attributes that can be used to perform the classification task.

These results are very promising, since disintegrating is a computationally expensive operation, but as Table 2 shows, we only need to disintegrate a small portion of the dataset for obtaining good results. Also, notice that when classifying new instances, we don't need to disintegrate them, just re-represent them, which is computationally much cheaper than disintegration. Re-representing simply means running one subsumption test for each of the different properties in the Taxonomic Vocabulary. 
Table 2

Performance of a nearest neighbor classifier with different sampling sizes $(\tau)$ for vocabulary creation; for each value of $\tau$ we report classification accuracy of three different distances and vocabulary size.

\begin{tabular}{|c||cc|c||cc|c|}
\hline \multicolumn{1}{|c||}{ Sample } & \multicolumn{3}{|c||}{ Soy (307) } & \multicolumn{3}{c||}{ Dem (280) } \\
\hline$\tau$ & Jaccard & Euclidean & Size & Jaccard & Euclidean & Size \\
\hline 0.01 & $86.97 \%$ & $86.32 \%$ & 92.04 & $88.57 \%$ & $87.50 \%$ & 95.39 \\
0.02 & $85.99 \%$ & $86.64 \%$ & 107.08 & $88.21 \%$ & $86.79 \%$ & 140.55 \\
0.05 & $90.55 \%$ & $90.88 \%$ & 122.00 & $93.21 \%$ & $90.71 \%$ & 197.80 \\
0.1 & $90.55 \%$ & $90.55 \%$ & 129.52 & $93.57 \%$ & $92.50 \%$ & 247.81 \\
0.2 & $90.88 \%$ & $90.88 \%$ & 133.11 & $93.93 \%$ & $93.93 \%$ & 306.56 \\
0.4 & $91.53 \%$ & $91.53 \%$ & 134.53 & $95.00 \%$ & $93.93 \%$ & 376.55 \\
0.6 & $90.88 \%$ & $90.88 \%$ & 134.82 & $95.36 \%$ & $93.57 \%$ & 428.26 \\
0.8 & $90.88 \%$ & $90.88 \%$ & 134.94 & $95.00 \%$ & $94.07 \%$ & 469.86 \\
1.0 & $91.21 \%$ & $91.21 \%$ & 135.00 & $95.00 \%$ & $93.47 \%$ & 506.39 \\
\hline Sample & \multicolumn{3}{||}{ Dem (503) } & \multicolumn{3}{c|}{ Trains (1000) } \\
\hline$\tau$ & Jaccard & Euclidean & Size & Jaccard & Euclidean & Size \\
\hline 0.01 & $83.50 \%$ & $83.10 \%$ & 149.81 & $92.00 \%$ & $91.40 \%$ & 86.68 \\
0.02 & $86.68 \%$ & $86.68 \%$ & 197.55 & $90.90 \%$ & $90.40 \%$ & 99.08 \\
0.05 & $89.46 \%$ & $88.87 \%$ & 271.67 & $91.40 \%$ & $91.20 \%$ & 116.42 \\
0.1 & $89.46 \%$ & $89.06 \%$ & 335.93 & $91.40 \%$ & $91.70 \%$ & 129.44 \\
0.2 & $90.86 \%$ & $89.46 \%$ & 408.60 & $91.40 \%$ & $91.20 \%$ & 141.11 \\
0.4 & $90.66 \%$ & $90.46 \%$ & 497.42 & $90.30 \%$ & $89.80 \%$ & 150.22 \\
0.6 & $90.66 \%$ & $90.06 \%$ & 560.37 & $91.20 \%$ & $90.30 \%$ & 154.40 \\
0.8 & $90.46 \%$ & $90.06 \%$ & 609.30 & $90.80 \%$ & $90.90 \%$ & 156.70 \\
1.0 & $90.66 \%$ & $89.26 \%$ & 651.61 & $90.70 \%$ & $90.40 \%$ & 158.00 \\
\hline
\end{tabular}

\section{Related Work}

Propositionalization in ILP transforms a relational representation of a learning problem into a propositional (feature-based, attribute-value) representation [12]. Clearly, propositionalization can be described as a re-representation that transforms examples into vectors of attribute-value pairs, while in our approach the re-representation transforms examples into sets of properties (multi-relational patterns, not attributevalue pairs).

An example of propositional learning techniques applied to relational data is adapting decision trees to ILP, e.g. the TILDE system [6]. TILDE presents a logical representation for decision trees and how to translate them into Prolog programs, and uses first order logic clauses to represent decisions (nodes) in the tree. MRDT (Multi-Relational Decision Tree Induction) were introduced in [11]. MRDT adds decision nodes to the tree through a process of successive refinement. MRDT defines a so-called selection graph where each node contains a multi-relational pattern, and a new split in the tree modifies ("refines") the selection graph.
While properties are also multi-relational patterns, they are defined by and obtained from the refinement graph of the generalization space, not a selection graph. Neither TILDE nor MRDT use re-representation of examples, and they focus on logical representations, while our approach can be used in any representation formalism where an adequate refinement graph could be defined.

\section{Conclusions}

This paper has introduced two key ideas. First we presented the idea of disintegration, which allows us to define a property-based vocabulary and to re-represent relational examples in this vocabulary. The second is that the E/P matrix $\mathbf{M}$ relating properties to examples can be used for any classical approach of propositional learning to relational data. The reason is that the E/P matrix $\mathbf{M}$ re-represents the relational examples into a Boolean vector of properties that each example satisfy or not. The only difference is that the values in that vector are relational patterns (properties) that can be obtained in a principled way after a refinement graph is specified. The E/P matrix $\mathbf{M}$ could be used for differ- 
$\left(\rho_{s}\right)$ SoRt SPeCialization:

$$
\left[\begin{array}{l}
s_{1}<s_{2} \wedge \nexists s: s_{1}<s<s_{2} \wedge \\
\forall X . f \doteq Y \in \phi, \exists s_{2} . f \doteq s_{3} \in O \wedge \\
\operatorname{sort}(Y) \geq s_{3}
\end{array}\right] \quad \frac{\phi \& X: s_{1}}{\phi \& X: s_{2}}
$$

$\left(\rho_{i}\right)$ Variable Introduction Without Sets:

$$
\left[\begin{array}{ll}
Y \in \mathcal{V} \wedge Y \notin \operatorname{var}(\phi) \wedge \\
s . f \doteq s^{\prime} \in O \wedge \\
f \notin \text { features }(X)
\end{array}\right] \quad \frac{\phi \& X: s}{\phi \& X: s \& X . f \doteq Y \& Y: s^{\prime}}
$$

$\left(\rho_{v}\right)$ VARIABLE INTRODUCTION:

$$
\left[\begin{array}{ll}
Y \in \mathcal{V} \wedge Y \notin \operatorname{var}(\phi) \wedge \\
s . f \doteq s^{\prime} \in O
\end{array}\right] \quad \frac{\phi \& X: s}{\phi \& X: s \& X . f \doteq Y \& Y: s^{\prime}}
$$

$\left(\rho_{n}\right)$ Non-Circular Variable Equality:

$$
\left[\begin{array}{l}
X, Y \in \operatorname{vars}(\psi) \wedge X \neq Y \wedge \\
\operatorname{sort}(X) \geq \operatorname{sort}(Y) \vee \operatorname{sort}(Y) \geq \operatorname{sort}(X) \wedge \\
X \notin \text { reachable }(Y) \wedge \\
Y \notin \text { reachable }(X)
\end{array}\right] \frac{\phi}{\phi \& X=Y}
$$

$\left(\rho_{e}\right)$ Variable Equality:

$$
\left[\begin{array}{l}
X, Y \in \operatorname{vars}(\psi) \wedge X \neq Y \wedge \\
\operatorname{sort}(X) \geq \operatorname{sort}(Y) \vee \operatorname{sort}(Y) \geq \operatorname{sort}(X)
\end{array}\right] \quad \frac{\phi}{\phi \& X=Y}
$$

Fig. 9. Specialization refinement operators, represented as rewriting rules.

ent purposes, like calculating the similarity among examples, using $\mathbf{M}$ to perform clustering, or finding the minimal set of properties that discriminate the positive examples belonging to a class with respect to the negative examples.

We presented experiments of using re-representation in the context of a nearest neighbor classifier. In our current work, we are developing property-based decision trees. One interesting aspect of our re-representation approach when used in conjunction with inductive machine learning techniques, such as decision trees, is taht once generalizations have been found over the rerepresentation, those can be mapped back to the original relational representation by using the integration operation.

Future work will also explore how to use the several distinct sublanguages (e.g. like the sublanguages of feature terms defined in Fig. 5) in machine learning techniques. The goal would be to find the simplest sub- language that fulfills the requirement of a specific ML technique for a given set of examples (following ideas from multi-strategy learning). Moreover, we intend to investigate the use of properties on other representation formalisms for which complete and locally finite refinement operators have been defined in the literature, such as ILP-restricted Horn Clauses [14] or some Description Logics [5].

\section{Appendix}

\section{A. Refinement Operators for Feature Terms}

In order to define languages of different expressiveness we will specify several different refinement operators. The collection of specialization refinement operators for feature terms are outlined in Figure 9 as rewrit- 
$\left(\gamma_{s}\right)$ SORT GeneralizATION:

$$
\left[\begin{array}{l}
s_{1}<s \wedge \nexists s_{2}: s_{1}<s_{2}<s \wedge \\
\forall X . f \doteq Y \in \phi \exists s_{1} \cdot f \doteq s_{3} \in O \wedge \\
\operatorname{sort}(Y) \geq s_{3}
\end{array}\right] \frac{\phi \& X: s}{\phi \& X: s_{1}}
$$

$\left(\gamma_{v}\right)$ VARIABLE ELIMINATION:

$$
\left[\begin{array}{l}
s . f \doteq s^{\prime} \in O \wedge \\
\text { features }(Y)=\emptyset
\end{array}\right] \quad \frac{\phi \& X: s \& X . f \doteq Y \& Y: s^{\prime}}{\phi \& X: s}
$$

$\left(\gamma_{e}\right)$ VARIABLE EQUALITy Elimination:

$$
\left[Z_{1} \notin \operatorname{vars}(\phi)\right] \quad \frac{\phi \& X . f \doteq Z \& Y . f^{\prime} \doteq Z}{\phi \& X . f \doteq Z \& Y . f^{\prime} \doteq Z_{1} \& Z_{1}: \operatorname{sort}(Z)}
$$

$\left(\gamma_{r}\right)$ RoOT VARIABLE EQUALITY ELIMINATION:

$$
\left[\begin{array}{l}
Z_{1} \notin \operatorname{vars}(\phi) \wedge \\
\operatorname{root}(\psi)=Z
\end{array}\right] \quad \frac{\phi \& X . f \doteq Z}{\phi \& X \cdot f \doteq Z_{1} \& Z_{1}: \operatorname{sort}(Z)}
$$

Fig. 10. Generalization operators for feature terms. Notice that this operators are not complete, but that they ensure reaching $\perp$ from any feature term in the language.

ing rules. A rewriting rule is composed of three parts: a top part, with the clause representation of a term, a lower part which represents the refinement of that term, and the applicability conditions of the rewrite rule (shown between square brackets in the left hand side of the definition). Let us briefly review each operator:

- $\rho_{s}$ generates refinements by substituting the sort $s_{1}$ of a variable in a term by a more specific sort $s_{2}$. Notice that the applicability condition ensures that $s_{1}$ is substituted only by a direct descendant sort $s_{2}$ (i.e. \#s $\in S: s_{1}<s<s_{2}$ ) while satisfying the restrictions in the ontology.

- $\rho_{i}$ generates refinements by adding a feature and its value to a term that previously didn't have that feature.

- $\rho_{v}$ generates refinements by adding a value to a feature: if the feature was undefined the operator will add the feature and its value or (if the feature was already defined) add a value to the a set of values of that feature.

- $\rho_{n}$ generates refinements by adding a non-circular variable equality.

- $\rho_{e}$ generates refinements by adding a variable equality (either circular or non circular).
The set of refinement operators $\left\{\rho_{s}, \rho_{v}, \rho_{e}\right\}$ for feature terms presented here is complete, finite and proper 2 . Notice that for other refinement graphs (such as the ones defined for description logics [16] or the ones defined by $\theta$-subsumption [13]) complete, finite and proper refinement operators do not exist ${ }^{3}$.

Figure 10 presents the generalization operators for feature terms. Notice that the generalization operators are the same regardless of the language of feature terms used. Nevertheless, depending of the language used, the operators will be complete or not. Concerning the generalization operator, it is not possible to define a complete and still locally finite operator when there are circular variable equalities. The operators shown in Figure 10 do ensure that $\perp$ is reached, but they are not complete. Specifically:

- $\gamma_{s}$ generalizes terms by substituting the sort of one of the variables in the term by a more general sort.

\footnotetext{
${ }^{2}$ The operators $\rho_{n}$ and $\rho_{e}$ shown in Figure 9 are a simplification that might generate some terms that are not refinements, but it's easy to filter those out using subsumption tests.

${ }^{3}$ The conditions identified by van der Laag and Nienhuys-Cheng [13] for proving the inexistence are not satisfied in our case, so the proof does not apply to our formalization.
} 
- $\gamma_{v}$ generalizes a term by removing the value of one of the features in one variable of the term. Notice that the operator only removes a variable $Y$ if features $(Y)=\emptyset$, i.e. if $Y$ has no defined features.

- $\gamma_{e}$ and $\gamma_{r}$ generalize a term by removing a variable equality. A circular variable equality can be removed in an infinite number of ways (see Appendix $\mathrm{C}$ ), and this is the cause of the generalization operators not being complete, although they still ensure that $\perp$ can be reached from any term.

Acknowledgements. Research partially supported by Projects Next-CBR (TIN2009-13692-C03-01) and Cognitio (TIN2012-38450- C03-03) and by the Generalitat de Catalunya under the grants 2009-SGR-1434.

\section{References}

[1] Aït-Kaci, H.: Description logic vs. order-sorted feature logic. In: Proc. 20th International Workshop on Description Logics. pp. 147-154 (2007)

[2] Aït-Kaci, H., Podelski, A.: Towards a meaning of LIFE. Tech. Rep. 11, Digital Research Laboratory (1992)

[3] Armengol, E., Plaza, E.: Relational case-based reasoning for carcinogenic activity prediction. Artif. Intell. Rev. 20(1-2), 121-141 (2003)

[4] Armengol, E., Plaza, E.: Lazy learning for predictive toxicology based on a chemical ontology. In: Dubitzky, W., Azuaje, F. (eds.) Artificial Intelligence Methods and Tools for Systems Biology, vol. 5, pp. 1-18. Springer-Verlag (2005)

[5] Badea, L., Nienhuys-Cheng, S.H.: A refinement operator for description logics. In: Cussens, J., Frisch, A. (eds.) Inductive Logic Programming. pp. 40-59. No. 1866 in Lecture Notes in Computer Science, Springer (1999)

[6] Blockeel, H., De Raedt, L.: Top-down induction of first-order logical decision trees. Artificial Intelligence 101, 285-297 (May 1998)

[7] Carpenter, B.: The Logic of Typed Feature Structures, Cambridge Tracts in Theoretical Computer Science, vol. 32. Cambridge University Press (1992)

[8] Emde, W., Wettschereck, D.: Relational instance based learning. In: Saitta, L. (ed.) Machine Learning - Proceedings 13th International Conference on Machine Learning. pp. 122 - 130. Morgan Kaufmann Publishers (1996)

[9] Falkenhainer, B., Forbus, K., Gentner, D.: The structure mapping engine: algorithm and examples. Artificial Intelligence 41, 1-63 (1990)

[10] Kashima, H., Tsuda, K., Inokuchi, A.: Marginalized kernels between labeled graphs. In: Proc. ICML'04. pp. 321-328 (2003)

[11] Knobbe, A.J., Siebes, A., Wallen, D.V.D., V, S.B.: Multirelational decision tree induction. In: Principles of Data Mining and Knowledge Discovery. pp. 378-383. Springer (1999)

[12] Kramer, S., Lavrač, N., Flach, P.: Propositionalization approaches to relational data mining. In: Džeroski, S., Lavrač, N. (eds.) Relational Data Mining, pp. 262-286. Springer (2000)
[13] van der Laag, P.R.J., Nienhuys-Cheng, S.H.: Existence and nonexistence of complete refinement operators. In: ECML-94: Proceedings of the European conference on machine learning on Machine Learning. pp. 307-322. Springer-Verlag New York, Inc., Secaucus, NJ, USA (1994)

[14] van der Laag, P.R.J., Nienhuys-Cheng, S.H.: Completeness and properness of refinement operators in inductive logic programming. J. Log. Program. 34(3), 201-225 (1998)

[15] Lavrač, N., Džeroski, S.: Inductive Logic Programming. Techniques and Applications. Ellis Horwood (1994)

[16] Lehmann, J., Hitzler, P.: Foundations of refinement operators for description logics. In: Blockeel, H., Shavlik, J.W., Tadepalli, P. (eds.) Proceedings of the 17th International Conference on Inductive Logic Programming (ILP). Lecture Notes in Computer Science, vol. 4894, pp. 161-174. Springer (2008)

[17] Michie, D., Muggleton, S., Page, D., Srinivasan, A.: To the international computing community: A new East-West challenge. Tech. rep., Oxford University Computing laboratory, Oxford,UK (1994)

[18] Ontañón, S., Plaza, E.: Similarity measures over refinement graphs. Machine Learning 87(1), 57-92 (Apr 2012)

[19] Sánchez, A., Ontañón, S., Calero, P.A.G., Plaza, E.: Measuring similarity in description logics using refinement operators. In: Ram, A., Wiratunga, N. (eds.) ICCBR'11: Proc. 19th International Conference on Case-Based Reasoning. Lecture Notes in Artificial Intelligence, vol. 6880, pp. 289 - 303 (2011)

[20] Sánchez-Ruiz-Granados, A.A., Ontañón, S., González-Calero, P.A., Plaza, E.: Refinement-based similarity measure over DL conjunctive queries. In: Delany, S.J., Ontañón, S. (eds.) ICCBR-13: Proc. 21st International Conference on Case-Based Reasoning. Lecture Notes in Computer Science, vol. 7969, pp. 270-284. Springer (2013) 